\title{
Existence of limit cycles for Liénard-type systems with $p$-Laplacian
}

\author{
Jitsuro SUGIE*, Ai KONO, Aya YAMAGUCHI \\ Department of Mathematics and Computer Science \\ Shimane University \\ Matsue 690-8504, Japan \\ e-mail: jsugie@riko.shimane-u.ac.jp
}

\begin{abstract}
The purpose of this paper is to give sufficient conditions under which an equivalent system to the equation $\left(\phi_{p}(\dot{x})\right)+f(x) \phi_{p}(\dot{x})+g(x)=0$ has at least one stable limit cycle, where $\phi_{p}(\cdot)$ is the one-dimensional $p$-Laplacian. The main results are proved by means of phase plane analysis with the Poincaré-Bendixson theorem. Sufficient conditions are also given for the origin $(x, \dot{x})=(0,0)$ to be unstable and for all solutions to be bounded in the future.

2000 Mathematics Subject Classification: 34C05, 34C07, 70K05, 70K42.

Key words: Limit cycles, phase plane analysis, one-dimensional $p$-Laplacian, Liénard system, Poincaré-Bendixson theorem, half-linear differential equations.
\end{abstract}

\section{Introduction}

In this paper, we deal with the generalized Liénard-type equation

$$
\left(\phi_{p}(\dot{x})\right)^{\bullet}+f(x) \phi_{p}(\dot{x})+g(x)=0
$$

and give some sufficient conditions for the existence of limit cycles of an equivalent system to (1.1). Here ${ }^{\circ}=d / d t ; \phi_{p}(y)$ is a real-valued function defined by

$$
\phi_{p}(y)=|y|^{p-2} y
$$

with $p>1$ a fixed real number; $f(x)$ and $g(x)$ satisfy suitable smoothness conditions for the uniqueness of solutions of initial value problem, for example, 
$f(x)$ is continuous on $\mathbb{R}$ and $g(x)$ is locally Lipschitz continuous on $\mathbb{R}$. Throughout this paper, we assume that

$$
x g(x)>0 \quad \text { if } x \neq 0 .
$$

Hence, the origin is the only critical point. Let

$$
G(x)=\int_{0}^{x} g(\xi) d \xi
$$

and denote by $G^{-1}(w)$ the inverse function of $w=G(x) \operatorname{sgn} x$.

Eq. (1.1) becomes the equation

$$
\left(\phi_{p}(\dot{x})\right)^{\cdot}+\alpha \phi_{p}(\dot{x})+\beta \phi_{p}(x)=0
$$

if $f(x)=\alpha \in \mathbb{R}$ and $g(x)=\beta \phi_{p}(x)$ with $\beta>0$. In the case $p=2$, Eq. (1.1) reduces to the classical Liénard equation

$$
\ddot{x}+f(x) \dot{x}+g(x)=0 .
$$

Let $x_{1}(t)$ and $x_{2}(t)$ be nontrivial solutions of (1.2). Then $c x_{1}(t)$ is also a solution of (1.2) for any $c \in \mathbb{R}$, but $x_{1}(t)+x_{2}(t)$ is not a solution of (1.2). For this reason, differential equations such as (1.2) is called "half-linear." Over the past four decades a considerable number of studies have been made on the oscillation of solutions of half-linear differential equations. For example, those results can be found in $[1,2,6,12]$. Although Eq. (1.2) may have an infinite number of periodic solutions, there are no limit cycles of the system

$$
\dot{x}=\phi_{p^{*}}(y), \quad \dot{y}=-\alpha y-\beta \phi_{p}(x)
$$

which is equivalent to (1.2). The situation is the same as planar autonomous linear systems. To see this, we take

$$
V(x, y)=\int_{0}^{y} \phi_{p^{*}}(\eta) d \eta+\beta \int_{0}^{x} \phi_{p}(\xi) d \xi
$$

as a Liapunov function (as to Liapunov's direct method, for example, see $[9,18]$ ). Then, calculating the derivative of $V$ along any solution of (1.4), we have $\dot{V}_{(1.4)}(x, y)=-\alpha|y|^{p^{*}}$. We therefore conclude that the global phase portrait of (1.4) is classified into three types according to $\alpha$ : (i) if $\alpha>0$, then all positive orbits approach the origin as $t$ increases; (ii) if $\alpha=0$, all positive orbits are closed curves surrounding the origin; (iii) if $\alpha<0$, then all positive orbits tend to $\infty$ as $t$ increases.

On the other hand, there is a strong possibility that an equivalent system to (1.3) has limit cycles. Indeed, we can find a great deal of literature concerning the existence of limit cycles for a system of the form

$$
\dot{x}=y, \quad \dot{y}=-f(x) y-g(x)
$$


under the standard assumptions:

$$
\begin{gathered}
f(0)<0 ; \\
f(x)>0 \quad \text { if }|x|>\delta
\end{gathered}
$$

for some $\delta>0$ (see $[3-5,8,10,13-17,19]$ and the references contained therein). As typical examples of those results, we can cite the following theorems which are obtained by Villari [15, 16] (see also [7, pp.101-104]).

Theorem A Under the assumptions (1.6) and (1.7), if

$$
\limsup _{x \rightarrow \infty} \frac{g(x)}{f(x)}<\infty \quad \text { or } \quad \liminf _{x \rightarrow-\infty} \frac{g(x)}{f(x)}>-\infty
$$

and there exist two numbers $h \geq \delta$ and $b>0$ such that

$$
f(x)+|g(x)|>b \quad \text { if }|x|>h,
$$

then system (1.5) has at least one stable limit cycle.

Theorem B Under the assumptions (1.6) and (1.7), if

$$
G(\infty)=G(-\infty)=\infty
$$

and there exist $\alpha<-\delta$ and $\beta>\delta$ such that

$$
\int_{\alpha}^{\beta} f(\xi) d \xi>0
$$

then system (1.5) has at least one stable limit cycle.

It is essential to find a bounded positive orbit of (1.5) in the proof of Theorems A and B. If such a positive orbit exists and the origin is locally repulsive (see Section 2 for the definition), then there is at least one stable limit cycle of (1.5) as a result of the Poincaré-Bendixson theorem. It follows from condition (1.6) that the origin is locally repulsive. Roughly speaking,

(i) in Theorem A, condition (1.8) implies that there is an unbounded negative orbit of (1.5) and conditions (1.7) and (1.9), in addition to (1.8), show that there is a bounded positive orbit of (1.5);

(ii) in Theorem B, conditions (1.7) and (1.10) imply that there is a positive orbit of (1.5) rotating around the origin clockwise and condition (1.11) indicates that the positive orbit is bounded.

It is only natural to consider the question of whether an equivalent system to (1.1) has at least one stable limit cycle under the conditions in Theorem A or B. What are more general conditions on $p, f(x)$ and $g(x)$ for the existence of limit 
cycles? However, there are few papers concerning limit cycles of an equivalent system to (1.1) (refer to [11]). We answer affirmatively to the question above and give some sufficient conditions which guarantee that an equivalent system to (1.1) has at least one stable limit cycle.

Our main theorems are stated in Section 4. To prove our results, we use phase plane analysis for Eq. (1.1) and the Poincaré-Bendixson theorem. In Section 2, we establish two kinds of criteria for the existence of a Poincaré-Bendixson domain. In Section 3, we present sufficient conditions under which the origin is unstable for Eq. (1.1). In Section 4, we examine the asymptotic behavior of solutions of (1.1) and give sufficient conditions for all solutions of (1.1) to be bounded in the future. The lemmas given in Sections 3 and 4 can be applied even to the half-linear differential equation (1.2).

\section{Poincaré-Bendixson domain}

Letting $y=\phi_{p}(\dot{x})$, we can rewrite Eq. (1.1) as the planar system

$$
\dot{x}=\phi_{p^{*}}(y), \quad \dot{y}=-f(x) y-g(x),
$$

where $p^{*}$ is the conjugate exponent of $p$ or the number satisfying

$$
\frac{1}{p}+\frac{1}{p^{*}}=1 \text {. }
$$

Note that $\phi_{p^{*}}(y)$ is the inverse function of $y=\phi_{p}(\dot{x})$. We denote by $\gamma^{+}(P)$ and $\gamma^{-}(P)$ the positive orbit and negative orbit of (2.1) starting at a point $P \in \mathbb{R}^{2}$, respectively. If an orbit of (2.1) crosses the $x$-axis, then its tangent is vertical at the point of intersection. In the study of the asymptotic behavior of solutions of (2.1), one of the most important point is whether all orbits intersect the $x$-axis or not.

Suppose that an orbit of (2.1) does not meet the $x$-axis. Then we may regard the orbit as a solution $y(x)$ of

$$
(p-1) \frac{d}{d x} \phi_{p^{*}}(y)=-f(x)-\frac{g(x)}{y},
$$

because

$$
|y|^{p^{*}-2} \frac{d y}{d x}=\frac{1}{p^{*}-1} \frac{d}{d x} \phi_{p^{*}}(y)=(p-1) \frac{d}{d x} \phi_{p^{*}}(y)
$$

by $(2.2)$.

For the sake of convenience, we say that system (2.1) has property $\left(X_{1}^{+}\right)$ (resp., $\left(X_{3}^{+}\right)$) if, for every point $P$ belonging to the first (resp., third) quadrant $Q_{1}$ (resp., $\left.Q_{3}\right), \gamma^{+}(P)$ crosses the $x$-axis. We also say that system (2.1) has property $\left(X_{2}^{-}\right)$(resp., $\left(X_{4}^{-}\right)$) if, for every point $P$ belonging to the second (resp., fourth) quadrant $Q_{2}$ (resp., $\left.Q_{4}\right), \gamma^{-}(P)$ crosses the $x$-axis. 
As is well known, the Poincaré-Bendixson theorem plays a major role in proving the existence of limit cycles for two-dimensional autonomous systems such as (2.1). To apply the Poincaré-Bendixson theorem, we have only to find a positively invariant set $S$ whose shape is a deformed annulus; that is, $S$ has an inner boundary and an outer boundary. We call $S$ a Poincaré-Bendixson domain. If system (2.1) has a Poincaré-Bendixson domain, then there exists at least one limit cycle in the domain. Note that the origin is always surrounded with the inner (outer) boundary of a Poincaré-Bendixson domain.

The origin is said to be locally repulsive if there is some neighborhood $U$ of it such that $\gamma^{+}(P)$ starting at any point $P \in U$ goes away from $U$ and $\gamma^{+}(P)$ starting at any point $P \in U^{c}$ does not enter into $U$, where $U^{c}$ is the complement of $U$. Hence, if the origin is locally repulsive, then system (2.1) has no limit cycle in $U$ and the complement $U^{c}$ is a positively invariant set.

We first clarify that properties $\left(X_{1}^{+}\right),\left(X_{2}^{-}\right),\left(X_{3}^{+}\right)$and $\left(X_{4}^{-}\right)$are fundamental concepts in constructing a positively invariant set with an outer boundary.

Lemma 2.1 Suppose that the origin is locally repulsive. If system (2.1) has properties $\left(X_{1}^{+}\right)$and $\left(X_{3}^{+}\right)$, but it fails to have property $\left(X_{2}^{-}\right)$or $\left(X_{4}^{-}\right)$, then a Poincaré-Bendixson domain exists.

Proof. We prove only the case that system (2.1) fails to have property $\left(X_{2}^{-}\right)$, because the proof of the other case is carried out in the same way. In this case, there exists a point $P_{0}=\left(x_{0}, y_{0}\right) \in Q_{2}$ such that $\gamma^{-}\left(P_{0}\right)$ runs to infinity without intersecting the negative $x$-axis. Let us examine the asymptotic behavior of $\gamma^{+}\left(P_{0}\right)$ hereafter.

We will show that $\gamma^{+}\left(P_{0}\right)$ meets the positive $y$-axis and then enters into $Q_{1}$. To this end, we prove that $\gamma^{+}\left(P_{0}\right)$ has no vertical asymptotes in the region $\left\{(x, y): x_{0}<x \leq 0\right.$ and $\left.y>0\right\}$. By way of contradiction, we suppose that $\gamma^{+}\left(P_{0}\right)$ has a vertical asymptotic line; that is, there exists an $x_{1}$ with $x_{0}<x_{1} \leq 0$ such that $\gamma^{+}\left(P_{0}\right)$ approaches the line $x=x_{1}$ and continues to rise until infinity. Let $y(x)$ be the solution of $(2.3)$ corresponding to $\gamma^{+}\left(P_{0}\right)$. Then we have

$$
y(x) \rightarrow \infty \quad \text { as } x \rightarrow x_{1} .
$$

Hence, there exist a $K>1$ and an $x_{2}$ with $x_{0}<x_{2}<x_{1}$ such that $y\left(x_{2}\right)=K$ and

$$
y(x)>K \text { for } x_{2}<x<x_{1} .
$$

Define $L=\max \left\{|f(x)|: x_{2} \leq x \leq x_{1}\right\}$ and $M=\max \left\{|g(x)|: x_{2} \leq x \leq x_{1}\right\}$. Then, noticing that $p^{*}>1$, we obtain

$$
\begin{aligned}
\frac{d}{d x} y(x) & =-f(x) y(x)^{2-p^{*}}-g(x) y(x)^{1-p^{*}} \\
& \leq|f(x)| y(x)+|g(x)| K^{1-p^{*}} \\
& \leq L y(x)+M K^{1-p^{*}}
\end{aligned}
$$


for $x_{2} \leq x \leq x_{1}$. From this inequality and (2.4), we get

$$
\infty=\int_{K}^{\infty} \frac{1}{L \eta+M K^{1-p^{*}}} d \eta \leq \int_{x_{2}}^{x_{1}} d \xi=x_{1}-x_{2}<\infty,
$$

which is a contradiction.

Let $P_{1}$ be the intersection point of $\gamma^{+}\left(P_{0}\right)$ with the positive $y$-axis. From the assumption that system (2.1) has property $\left(X_{1}^{+}\right)$it follows that $\gamma^{+}\left(P_{0}\right)$ passes through $Q_{1}$ until it meets the positive $x$-axis. By the same manner as in the preceding paragraph, we can show that $\gamma^{+}\left(P_{0}\right)$ has no vertical asymptotes in $Q_{4}$. Hence, $\gamma^{+}\left(P_{0}\right)$ enters into $Q_{3}$, and therefore, it crosses the negative $x$-axis because system (2.1) has property $\left(X_{3}^{+}\right)$. From the uniqueness of solutions of (2.1), we see that $\gamma^{+}\left(P_{0}\right)$ does not intersect $\gamma^{-}\left(P_{0}\right)$. Hence, $\gamma^{+}\left(P_{0}\right)$ returns to the positive $y$-axis at a point $P_{2}$ which lies below $P_{1}$.

We denote by $R$ the region that is enclosed by the $\operatorname{arc} P_{1} P_{2}$ of $\gamma^{+}\left(P_{0}\right)$ and the line segment $P_{1} P_{2}$. Then $R$ is a positively invariant set. Since the origin is locally repulsive, we can find a neighborhood $U$ of the origin such that $U^{c}$ is another positively invariant set. Needless to say, $R$ contains $U$. Hence, the deformed annulus $R \backslash U$ is a Poincaré-Bendixson domain. This completes the proof of Lemma 2.1.

In case system (2.1) has properties $\left(X_{2}^{-}\right)$and $\left(X_{4}^{-}\right)$, we cannot always compose a positively invariant set with an outer boundary even if system (2.1) has properties $\left(X_{1}^{+}\right)$and $\left(X_{3}^{+}\right)$. For example, we consider the case that $p=2$, $f(x) \equiv-1$ and $g(x)=x$. Then system (2.1) becomes

$$
\dot{x}=y, \quad \dot{y}=y-x .
$$

The global phase portrait is a family of spirals and every positive orbit tends away from the origin as $t$ increases. In this case, system (2.1) has properties $\left(X_{1}^{+}\right),\left(X_{2}^{-}\right),\left(X_{3}^{+}\right)$and $\left(X_{4}^{-}\right)$, but it has no positively invariant set with an outer boundary. Thus, if we do not assume that system (2.1) fails to have property $\left(X_{2}^{-}\right)$or $\left(X_{4}^{-}\right)$, then we need another assumption for the existence of a positively invariant set with an outer boundary.

To present such an assumption, we consider an auxiliary system

$$
\dot{x}=\phi_{p^{*}}(y), \quad \dot{y}=-\tilde{f}(x) y-g(x),
$$

where $\tilde{f}(x)$ is defined by $\tilde{f}(x)=f(x)$ for $x<0, \tilde{f}(0)=0$ and

$$
\tilde{f}\left(G^{-1}(w)\right)=\frac{g\left(G^{-1}(w)\right)}{g\left(G^{-1}(-w)\right)} f\left(G^{-1}(-w)\right) \quad \text { for } w>0 .
$$

If $g(x)$ is an odd function, then $\tilde{f}(x)$ is also an odd function. In this case, all orbits of (2.5) are completely symmetrical with respect to the $y$-axis. Note that $\tilde{f}(x)$ may not be continuous at $x=0$, and therefore, orbits of (2.5) make a turn on the $y$-axis. 
Lemma 2.2 All orbits of (2.5) are deformed symmetrical with respect to the $y$-axis.

Proof. Changing variables

$$
u=\sqrt{2 G(x)} \operatorname{sgn} x, \quad v=y, \quad \frac{d \tau}{d t}=\frac{g(x) \operatorname{sgn} x}{\sqrt{2 G(x)}}=\frac{g(x)}{u}
$$

and denoting $\tau$ by $t$ again, we can transform system (2.5) into the system

$$
\dot{u}=\phi_{p^{*}}(v), \quad \dot{v}=-f_{1}(u) v-u,
$$

where

$$
f_{1}(u)=\frac{\tilde{f}\left(G^{-1}\left(u^{2} / 2 \operatorname{sgn} u\right)\right)}{g\left(G^{-1}\left(u^{2} / 2 \operatorname{sgn} u\right)\right)} u .
$$

The mapping $(x, y) \mapsto(u, v)$ is a homeomorphism of the $(x, y)$-plane onto the region $\{(u, v):-\sqrt{2 G(-\infty)}<u<\sqrt{2 G(\infty)}$ and $y \in \mathbb{R}\}$ and the correspondence between all orbits of (2.5) and those of (2.7) is one to one. Since $w=G(x) \operatorname{sgn} x=$ $u^{2} / 2 \operatorname{sgn} u$, we rewrite $(2.8)$ as

$$
f_{1}(u)=\frac{\tilde{f}\left(G^{-1}(w)\right)}{g\left(G^{-1}(w)\right)} u
$$

From the definition of $\tilde{f}(x)$, we see that

$$
\frac{\tilde{f}\left(G^{-1}(w)\right)}{g\left(G^{-1}(w)\right)}=\frac{f\left(G^{-1}(-w)\right)}{g\left(G^{-1}(-w)\right)}=\frac{\tilde{f}\left(G^{-1}(-w)\right)}{g\left(G^{-1}(-w)\right)} \quad \text { for } w>0,
$$

which shows that $\tilde{f}\left(G^{-1}(w)\right) / g\left(G^{-1}(w)\right)$ is an even function of $w$. Hence, the function $f_{1}(u)$ satisfies

$$
\begin{aligned}
f_{1}(-u) & =\frac{\tilde{f}\left(G^{-1}(-w)\right)}{g\left(G^{-1}(-w)\right)}(-u) \\
& =-\frac{\tilde{f}\left(G^{-1}(w)\right)}{g\left(G^{-1}(w)\right)} u=-f_{1}(u) ;
\end{aligned}
$$

that is, it is an odd function of $u$. Taking account of the vector field of (2.7), we see that all orbits have mirror symmetry about the $y$-axis. Thus, all orbits of (2.5) are deformed symmetrical with respect to the $y$-axis. The lemma is proved.

Comparing positive orbits of (2.1) and (2.5) starting at the same point, we have the following result. 
Lemma 2.3 Suppose that the origin is locally repulsive and that there exists a $w_{0}>0$ such that

$$
\begin{aligned}
& \frac{f\left(G^{-1}(-w)\right)}{g\left(G^{-1}(-w)\right)} \leq \frac{f\left(G^{-1}(w)\right)}{g\left(G^{-1}(w)\right)} \quad \text { for } w>w_{0} \\
& \int_{0}^{w_{0}}\left\{\frac{f\left(G^{-1}(w)\right)}{g\left(G^{-1}(w)\right)}-\frac{f\left(G^{-1}(-w)\right)}{g\left(G^{-1}(-w)\right)}\right\} d w>0 .
\end{aligned}
$$

If system (2.1) has properties $\left(X_{1}^{+}\right)$and $\left(X_{3}^{+}\right)$, then a Poincaré-Bendixson domain exists.

Remark 2.4 Conditions (2.9) and (2.10) do not require that $f(x)>0$ for $|x|$ sufficiently large. These conditions are available even in the case that $f(x)<0$ for $x>0$ or $x<0,|x|$ sufficiently large.

Proof of Lemma 2.3. As a consequence of Lemma 2.1, the lemma is true if system (2.1) fails to have property $\left(X_{2}^{-}\right)$or $\left(X_{4}^{-}\right)$. Hence, we consider only the case that system (2.1) has properties $\left(X_{2}^{-}\right)$and $\left(X_{4}^{-}\right)$. Since $\tilde{f}(x)=f(x)$ for $x<0$, system (2.5) coincides with system (2.1) in the left-half plane, and therefore, system (2.5) also has properties $\left(X_{2}^{-}\right)$and $\left(X_{3}^{+}\right)$. Lemma 2.2 asserts that all orbits of $(2.5)$ are in the deformed symmetry about the $y$-axis. From this fact, we see that system (2.5) has properties $\left(X_{1}^{+}\right)$and $\left(X_{4}^{-}\right)$.

By (2.6) and (2.9), we have

$$
\frac{\tilde{f}\left(G^{-1}(w)\right)}{g\left(G^{-1}(w)\right)}=\frac{f\left(G^{-1}(-w)\right)}{g\left(G^{-1}(-w)\right)} \leq \frac{f\left(G^{-1}(w)\right)}{g\left(G^{-1}(w)\right)} \quad \text { for } w>w_{0} .
$$

Hence, we obtain

$$
\tilde{f}\left(G^{-1}(w)\right) \leq f\left(G^{-1}(w)\right) \text { for } w>w_{0}
$$

namely,

$$
\tilde{f}(x) \leq f(x) \text { for } x>x_{0} \stackrel{\text { def }}{=} G^{-1}\left(w_{0}\right) .
$$

Also, it follows from (2.10) that

$$
\int_{0}^{w_{0}}\left\{\frac{f\left(G^{-1}(w)\right)}{g\left(G^{-1}(w)\right)}-\frac{\tilde{f}\left(G^{-1}(w)\right)}{g\left(G^{-1}(w)\right)}\right\} d w>0 .
$$

Put $x=G^{-1}(w)$. Then we get

$$
\int_{0}^{x_{0}}\{f(x)-\tilde{f}(x)\} d x>0 .
$$

For simplicity, we denote by $\rho$ the above definite integral. 
Define $L=\max \left\{|f(x)|: 0 \leq x \leq x_{0}\right\}$ and $M=\max \left\{g(x): 0 \leq x \leq x_{0}\right\}$. We choose two positive numbers $N$ and $K$ such that

$$
\begin{gathered}
M x_{0}<N \rho \\
K>\left\{N^{p^{*}-1}+\left(p^{*}-1\right)\left(L+\frac{M}{N}\right) x_{0}\right\}^{p-1} .
\end{gathered}
$$

Consider the rectangle $\left\{(x, y): 0 \leq x \leq x_{0}\right.$ and $\left.|y| \leq K\right\}$. Since system (2.5) has properties $\left(X_{1}^{+}\right),\left(X_{2}^{-}\right),\left(X_{3}^{+}\right)$and $\left(X_{4}^{-}\right)$, we can find a closed orbit of $(2.5)$ surrounding the rectangle. Let $P_{1}, P_{2}$ and $P_{3}$ be intersecting points of the closed orbit with the positive $y$-axis, the half line $l=\left\{(x, y): x=x_{0}\right.$ and $\left.y<0\right\}$ and the negative $y$-axis, respectively. We denote by $\tilde{\gamma}^{+}\left(P_{1}\right)$ the closed orbit of $(2.5)$.

To compare with $\tilde{\gamma}^{+}\left(P_{1}\right)$, we consider the positive orbit $\gamma^{+}\left(P_{1}\right)$ of $(2.1)$. As long as $\gamma^{+}\left(P_{1}\right)$ is in $Q_{1}$, we may regard $\gamma^{+}\left(P_{1}\right)$ as the solution $y(x)$ of $(2.3)$ satisfying $(0, y(0))=P_{1}$. Similarly, we may consider $\tilde{\gamma}^{+}\left(P_{1}\right)$ as the solution $\tilde{y}(x)$ of

$$
(p-1) \frac{d}{d x} \phi_{p^{*}}(y)=-\tilde{f}(x)-\frac{g(x)}{y}
$$

satisfying $(0, \tilde{y}(0))=P_{1}$ as long as $\tilde{\gamma}^{+}\left(P_{1}\right)$ is in $Q_{1}$. As in the proof of Lemma 2.1, we see that $\gamma^{+}\left(P_{1}\right)$ has no vertical asymptotes.

Claim 1. $y(x)>N$ for $0 \leq x \leq x_{0}$.

Suppose that the claim is not true; that is, there exists an $x_{1}$ with $0<x_{1} \leq$ $x_{0}$ such that $y\left(x_{1}\right)=N$ and $y(x)>N$ for $0 \leq x<x_{1}$. Then we have

$$
(p-1) \frac{d}{d x} \phi_{p^{*}}(y(x))=-f(x)-\frac{g(x)}{y(x)} \geq-L-\frac{M}{N}
$$

for $0 \leq x \leq x_{1}$. Integrating this inequality from 0 to $x_{1}$, we get

$$
(p-1)\left\{\phi_{p^{*}}\left(y\left(x_{1}\right)\right)-\phi_{p^{*}}(y(0))\right\} \geq-\left(L+\frac{M}{N}\right) x_{1} \geq-\left(L+\frac{M}{N}\right) x_{0} .
$$

Since $y\left(x_{1}\right)=N$ and $y(0)>K$, we obtain

$$
(p-1) N^{p^{*}-1}+\left(L+\frac{M}{N}\right) x_{0} \geq(p-1) \phi_{p^{*}}(y(0))>(p-1) \phi_{p^{*}}(K)=(p-1) K^{p^{*}-1} .
$$

This is a contradiction to (2.13).

Claim 2. $\tilde{y}\left(x_{0}\right)>y\left(x_{0}\right)$.

It follows from Claim 1 that

$$
(p-1) \frac{d}{d x} \phi_{p^{*}}(y(x))=-f(x)-\frac{g(x)}{y(x)} \leq-f(x)
$$


for $0 \leq x \leq x_{0}$. Integrate both sides to obtain

$$
(p-1)\left\{\phi_{p^{*}}\left(y\left(x_{0}\right)\right)-\phi_{p^{*}}(y(0))\right\} \leq-\int_{0}^{x_{0}} f(x) d x .
$$

Since $\tilde{\gamma}^{+}\left(P_{1}\right)$ surrounds the rectangle $\left\{(x, y): 0 \leq x \leq x_{0}\right.$ and $\left.|y| \leq K\right\}$, we see that $\tilde{y}(x)>K>N$ for $0 \leq x \leq x_{0}$. Hence, by (2.14) we have

$$
(p-1) \frac{d}{d x} \phi_{p^{*}}(\tilde{y}(x))=-\tilde{f}(x)-\frac{g(x)}{\tilde{y}(x)} \geq-\tilde{f}(x)-\frac{M}{N}
$$

for $0 \leq x \leq x_{0}$. This yields that

$$
(p-1)\left\{\phi_{p^{*}}\left(\tilde{y}\left(x_{0}\right)\right)-\phi_{p^{*}}(\tilde{y}(0))\right\} \geq-\int_{0}^{x_{0}} \tilde{f}(x) d x-\frac{M}{N} x_{0} .
$$

Note that $y(0)=\tilde{y}(0)$. Then, by (2.12), (2.15) and (2.16) we obtain

$$
\begin{aligned}
(p-1)\left\{\phi_{p^{*}}\left(\tilde{y}\left(x_{0}\right)\right)-\phi_{p^{*}}(y(0))\right\} & \geq \int_{0}^{x_{0}}\{f(x)-\tilde{f}(x)\} d x-\frac{M}{N} x_{0}-\int_{0}^{x_{0}} f(x) d x \\
& =\rho-\frac{M}{N} x_{0}-\int_{0}^{x_{0}} f(x) d x>-\int_{0}^{x_{0}} f(x) d x \\
& \geq(p-1)\left\{\phi_{p^{*}}\left(y\left(x_{0}\right)\right)-\phi_{p^{*}}(y(0))\right\} .
\end{aligned}
$$

We therefore conclude that $\phi_{p^{*}}\left(\tilde{y}\left(x_{0}\right)\right)>\phi_{p^{*}}\left(y\left(x_{0}\right)\right)$, namely, $\tilde{y}\left(x_{0}\right)>y\left(x_{0}\right)$.

Compare the vector field of (2.1) with that of (2.5) in the region $\{(x, y): x \geq$ $x_{0}$ and $\left.y \in \mathbb{R}\right\}$. Then, from (2.11) and Claim 2, we see that $\gamma^{+}\left(P_{1}\right)$ does not meet $\tilde{\gamma}^{+}\left(P_{1}\right)$, and therefore, it crosses the half line $l$ at a point which lies above $P_{2}$.

Next, we consider the positive orbit $\gamma^{+}\left(P_{2}\right)$ of (2.1) and compare it with $\tilde{\gamma}^{+}\left(P_{1}\right)$. Recall that $\tilde{\gamma}^{+}\left(P_{1}\right)$ passes through $P_{2}$. In much the same manner as Claims 1 and 2, we can show that

(i) $\gamma^{+}\left(P_{2}\right)$ does not meet the line segment $\left\{(x, y): 0 \leq x \leq x_{0}\right.$ and $\left.y=-N\right\}$;

(ii) $\gamma^{+}\left(P_{2}\right)$ crosses the negative $y$-axis at a point which lies above $P_{3}$.

System (2.1) coincides with system (2.5) in the left-half plane. Hence, by the uniqueness of solutions of $(2.1)$, we see that $\gamma^{+}\left(P_{2}\right)$ does not intersect $\tilde{\gamma}^{+}\left(P_{1}\right)$. This means that $\gamma^{+}\left(P_{2}\right)$ goes through $Q_{3}$ and $Q_{2}$, and then it meets the positive $y$-axis at a point which lies below $P_{1}$.

From the uniqueness of solutions of $(2.1)$ and the above properties of $\gamma^{+}\left(P_{1}\right)$ and $\gamma^{+}\left(P_{2}\right)$, it turns out that $\gamma^{+}\left(P_{1}\right)$ goes around the origin in clockwise order and returns the positive $y$-axis at a point $P_{4}$ which lies below $P_{1}$. We denote by $R$ the region that is enclosed by the arc $P_{1} P_{4}$ of $\gamma^{+}\left(P_{1}\right)$ and the line segment $P_{1} P_{4}$. Then $R$ is a positively invariant set. Since the origin is locally repulsive, we can produce a Poincaré-Bendixson domain whose outer boundary is $\partial R$. We have thus proved the lemma. 


\section{Repulsiveness of the origin}

In this section, we give sufficient conditions for the origin of (2.1) to be locally repulsive. If there exists a point $P$ on the positive (resp., negative) $x$-axis such that $\gamma^{+}(P)$ approaches the origin through only $Q_{4}$ (resp., $Q_{2}$ ), then the origin is not locally repulsive. For this reason, we have to examine the asymptotic behavior of $\gamma^{+}(P)$ for each point $P$ on the $x$-axis in a neighborhood of the origin.

To begin with, we transform system (2.1) into a standard type. Let

$$
\phi_{p}(u)=(p G(x))^{1 / p^{*}} \operatorname{sgn} x
$$

and $v=y$. Then $x=G^{-1}\left(\left(\phi_{p}(|u|)\right)^{p^{*}} / p \operatorname{sgn} u\right)$ and system $(2.1)$ is transferred into

$$
\frac{d u}{d \tau}=\phi_{p^{*}}(v), \quad \frac{d v}{d \tau}=-f_{2}(u) v-\phi_{p}(u)
$$

in which $d \tau / d t=g(x) \operatorname{sgn} x /(p G(x))^{1 / p^{*}}=g(x) / \phi_{p}(u)$ and

$$
f_{2}(u)=\frac{f(x)}{g(x)} \phi_{p}(u)=\frac{f(x)}{|g(x)|}(p G(x))^{1 / p^{*}} .
$$

For simplicity, we denote $\tau$ by $t$ again. Then we have

$$
\dot{u}=\phi_{p^{*}}(v), \quad \dot{v}=-f_{2}(u) v-\phi_{p}(u) .
$$

By virtue of the one-to-one correspondence between all orbits of (2.1) and those of (3.1), we can prove the following result.

Lemma 3.1 If there exists a $\lambda<p$ such that

$$
\frac{f(x)}{|g(x)|}(p G(x))^{1 / p^{*}} \leq \lambda
$$

for $|x|>0$ sufficiently small, then

(i) for every point $P$ on the positive $x$-axis, $\gamma^{+}(P)$ of $(2.1)$ crosses the negative $y$-axis, namely, it does not approach the origin through only $Q_{4}$;

(ii) for every point $P$ on the negative $x$-axis, $\gamma^{+}(P)$ of $(2.1)$ crosses the positive $y$-axis, namely, it does not approach the origin through only $Q_{2}$.

Proof. We will prove only the first statement, because we can use the same argument in the proof of the second statement.

We have only to show that any positive orbit of (3.1) starting at a point on the positive $u$-axis crosses the negative $v$-axis. The proof is by contradiction. Suppose that for $u_{0}>0$ sufficiently small, the positive orbit of (3.1) starting 
at $\left(u_{0}, 0\right)$ approaches the origin through only $Q_{4}$. From (3.2), we may assume without loss of generality that

$$
f_{2}(u) \leq \lambda \text { for } 0<u \leq u_{0} .
$$

Let $P_{0}=\left(u_{0}, 0\right)$ and we call this positive orbit $\gamma^{+}\left(P_{0}\right)$. Then we can regard $\gamma^{+}\left(P_{0}\right)$ as the solution $v(u)$ of

$$
(p-1) \frac{d}{d u} \phi_{p^{*}}(v)=-f_{2}(u)-\frac{\phi_{p}(u)}{v}
$$

satisfying $v\left(u_{0}\right)=0$.

For a parameter $k \geq 0$, let

$$
C_{k}=\left\{(u, v): u>0 \text { and } v=-\phi_{p}(k u)\right\} .
$$

Note that the curves $C_{0}$ and $C_{\infty}$ correspond to the positive $u$-axis and the negative $v$-axis, respectively, and

$$
\bigcup_{k=0}^{\infty} C_{k}=Q_{4}
$$

Define the function $W(u, v)$ by

$$
W(u, v)=k \quad \text { if }(u, v) \in C_{k} .
$$

By a simple calculation, we obtain the inequality

$$
\lambda<p \leq(p-1) k+\left(\frac{1}{k}\right)^{p-1}
$$

for all $k>0$. Hence, together with (3.3), we have

$$
\begin{aligned}
(p-1) \frac{d}{d u} \phi_{p^{*}}\left(-\phi_{p}(k u)\right) & =-(p-1) k \leq-p+\left(\frac{1}{k}\right)^{p-1} \\
& <-f_{2}(u)+\left(\frac{1}{k}\right)^{p-1} \\
& =-f_{2}(u)-\frac{\phi_{p}(u)}{-\phi_{p}(k u)}
\end{aligned}
$$

for $0<u \leq u_{0}$. This means that the slope of $C_{k}$ is smaller than that of $\gamma^{+}\left(P_{0}\right)$ at any point on $C_{k}$. In other words, $\gamma^{+}\left(P_{0}\right)$ moves from the region $\{(u, v): 0<$ $u \leq u_{0}$ and $\left.-\phi_{p}(k u)<v<0\right\}$ into the region $\left\{(u, v): 0<u \leq u_{0}\right.$ and $v<$ $\left.-\phi_{p}(k u)\right\}$ and then it does not cross the curve $C_{k}$ again. We therefore conclude that $W(u, v(u))$ becomes larger as $u$ decreases.

We will show that

$$
W(u, v(u)) \rightarrow \infty \quad \text { as } u \rightarrow 0 .
$$


If $W(u, v(u))$ does not diverge to $\infty$ as $u \rightarrow 0$, then there exists a $k^{*}>0$ such that

$$
W(u, v(u)) \nearrow k^{*} \quad \text { as } u \rightarrow 0 .
$$

Because of $(3.5)$, we can choose an $\varepsilon>0$ so that

$$
\lambda<(p-1)\left(k^{*}-\varepsilon\right)+\left(\frac{1}{k^{*}}\right)^{p-1} .
$$

By (3.6), there exists a $u_{1}$ with $0<u_{1}<u_{0}$ such that $W\left(u_{1}, v\left(u_{1}\right)\right)=k^{*}-\varepsilon$ and

$$
k^{*}-\varepsilon<W(u, v(u))<k^{*} \text { for } 0<u<u_{1},
$$

namely, $v\left(u_{1}\right)=-\phi_{p}\left(\left(k^{*}-\varepsilon\right) u_{1}\right)$ and

$$
-\phi_{p}\left(k^{*} u\right)<v(u)<-\phi_{p}\left(\left(k^{*}-\varepsilon\right) u\right) \text { for } 0<u<u_{1} .
$$

Hence, it follows from (3.4) that

$$
\begin{aligned}
-(p-1) k^{*} u+(p-1)\left(k^{*}-\varepsilon\right) u_{1} & <(p-1) \phi_{p^{*}}(v(u))-(p-1) \phi_{p^{*}}\left(v\left(u_{1}\right)\right) \\
& =-\int_{u_{1}}^{u} f_{2}(\xi) d \xi-\int_{u_{1}}^{u} \frac{\phi_{p}(\xi)}{v(\xi)} d \xi \\
& <-\lambda\left(u-u_{1}\right)-\int_{u_{1}}^{u} \frac{\phi_{p}(\xi)}{-\phi_{p}\left(k^{*} \xi\right)} d \xi \\
& =\lambda\left(u_{1}-u\right)-\left(\frac{1}{k^{*}}\right)^{p-1}\left(u_{1}-u\right)
\end{aligned}
$$

for $0<u<u_{1}$. On account of (3.7), however, this inequality fails to hold for a sufficiently small $u>0$. This is a contradiction.

From the divergence of $W(u, v(u))$ it turns out that for any $k>\lambda /(p-1)$, there exists a $u_{2}$ with $0<u_{2}<u_{0}$ such that

$$
v(u)<-\phi_{p}(k u)<0 \text { for } 0<u \leq u_{2} .
$$

Since $v(u)<0<\phi_{p}(u)$ for $0<u \leq u_{2}$, it follows from (3.3) and (3.4) that

$$
(p-1) \frac{d}{d u} \phi_{p^{*}}(v(u))=-f_{2}(u)-\frac{\phi_{p}(u)}{v(u)}>-\lambda
$$

for $0<u_{2} \leq u$. Integrating this inequality from 0 to $u_{2}$ and using (3.8), we have

$$
\begin{aligned}
-\lambda u_{2} & <(p-1) \phi_{p^{*}}\left(v\left(u_{2}\right)\right) \\
& <(p-1) \phi_{p^{*}}\left(-\phi_{p}\left(k u_{2}\right)\right) \\
& =-(p-1) k u_{2} .
\end{aligned}
$$


Since $u_{2}>0$ and $k>\lambda /(p-1)$, we obtain

$$
\lambda>(p-1) k>\lambda .
$$

This is a contradiction. Thus, any positive orbit of (3.1) starting at a point on the positive $u$-axis crosses the negative $v$-axis, thereby completing the proof of Lemma 3.1.

By means of Lemma 3.1, we give sufficient conditions under which the origin of (2.1) is locally repulsive as follows.

Lemma 3.2 Assume (3.2). If there exists a $\delta_{0}>0$ such that

$$
\frac{f\left(G^{-1}(-w)\right)}{g\left(G^{-1}(-w)\right)} \geq \frac{f\left(G^{-1}(w)\right)}{g\left(G^{-1}(w)\right)} \quad \text { but } \quad \frac{f\left(G^{-1}(-w)\right)}{g\left(G^{-1}(-w)\right)} \not \equiv \frac{f\left(G^{-1}(w)\right)}{g\left(G^{-1}(w)\right)}
$$

for $0<w<\delta_{0}$, then the origin of (2.1) is locally repulsive.

Remark 3.3 It is obvious that $g\left(G^{-1}(-w)\right)<0<g\left(G^{-1}(w)\right)$ for all $w>0$. If $f(0)<0$, then there exists a $\delta_{0}>0$ such that $f\left(G^{-1}(-w)\right)<0$ and $f\left(G^{-1}(w)\right)<0$ for $0<w<\delta_{0}$. Hence, condition (3.9) is satisfied. Condition (3.9) is applicable even in the case that $f(0)=0$. Since the origin of (2.1) is stable in the case that $f(0)>0$, it is quite natural that condition (3.9) does not hold in this case.

Proof of Lemma 3.2. Consider system (2.5) again. We will compare an orbit of (2.1) with an orbit of (2.5) starting at the same point. Recall that the function $\tilde{f}(x)$ in system (2.5) satisfies $\tilde{f}(x)=f(x)$ for $x<0, \tilde{f}(0)=0$ and condition $(2.6)$. By (2.6) and (3.9), we have

$$
\frac{\tilde{f}\left(G^{-1}(w)\right)}{g\left(G^{-1}(w)\right)} \geq \frac{f\left(G^{-1}(w)\right)}{g\left(G^{-1}(w)\right)} \quad \text { but } \quad \frac{\tilde{f}\left(G^{-1}(w)\right)}{g\left(G^{-1}(w)\right)} \not \equiv \frac{f\left(G^{-1}(w)\right)}{g\left(G^{-1}(w)\right)}
$$

for $0<w<\delta_{0}$, and therefore, we obtain

$$
\tilde{f}(x) \geq f(x) \text { but } \tilde{f}(x) \not \equiv f(x) \text { for } 0<x<\varepsilon_{0} \stackrel{\text { def }}{=} G^{-1}\left(\delta_{0}\right) .
$$

By the assumption that (3.2) is satisfied for $|x|>0$ sufficiently small, it follows from Lemma 3.1 that system (2.1) has properties

(i) every positive orbit starting at a point on the positive $x$-axis passes through only $Q_{4}$ and crosses the negative $y$-axis;

(ii) every positive orbit starting at a point on the negative $x$-axis passes through only $Q_{2}$ and crosses the positive $y$-axis. 
Since $\tilde{f}(x)=f(x)$ for $x<0$, system (2.5) coincides with system (2.1) in the left-half plane. Hence, system (2.5) also have the above property (ii). As shown in Lemma 2.2, all orbits of (2.5) are deformed symmetrical with respect to the $y$-axis. From this symmetry, we see that every negative orbit starting at a point on the positive $x$-axis passes through only $Q_{1}$ and crosses the positive $y$-axis.

Let us now consider an orbit of (2.5) passing through a point $P_{0}=\left(x_{0}, 0\right)$ with $0<x_{0}<\varepsilon_{0}$. From the above-mentioned reason it turns out that $\tilde{\gamma}^{-}\left(P_{0}\right)$ of (2.5) goes through the upper-half plane and meets the negative $x$-axis. Let $P_{1}$ be the intersection point of $\tilde{\gamma}^{-}\left(P_{0}\right)$ with the negative $x$-axis. Also, we see that $\tilde{\gamma}^{-}\left(P_{0}\right)$ enters into $Q_{3}$ and then it crosses the negative $y$-axis at a point or approaches the origin as $t$ decreases. By the symmetry of orbits of $(2.5), \tilde{\gamma}^{+}\left(P_{0}\right)$ crosses the negative $y$-axis at a point or tends to the origin as $t$ increases. In the former case, $\tilde{\gamma}^{+}\left(P_{0}\right)$ is a simple closed loop surrounding the origin. On the other hand, in the latter case, a closed curve is made by $\tilde{\gamma}^{+}\left(P_{0}\right), \tilde{\gamma}^{-}\left(P_{0}\right)$ and the origin.

Comparing the vector field of (2.1) with that of (2.5) in the upper-half plane, we see that $\gamma^{-}\left(P_{0}\right)$ of $(2.1)$ does not cross the arc $P_{0} P_{1}$ of $\tilde{\gamma}^{-}\left(P_{0}\right)$ because $(3.10)$ holds and $\tilde{f}(x)=f(x)$ for $x<0$. Hence, there are two possibilities that

(a) $\gamma^{-}\left(P_{0}\right)$ meets the negative $x$-axis at a point $P_{2}$ which is on the right-hand side of $P_{1}$;

(b) $\gamma^{-}\left(P_{0}\right)$ approaches the origin as $t$ decreases.

We next observe the asymptotic behavior of $\gamma^{+}\left(P_{0}\right)$ of (2.1). Since system (2.1) has the above property $(\mathrm{i}), \gamma^{+}\left(P_{0}\right)$ passes through only $Q_{4}$ and crosses the negative $y$-axis. From (3.10) again, we see that $\gamma^{+}\left(P_{0}\right)$ does not intersect $\tilde{\gamma}^{+}\left(P_{0}\right)$. To be exact, $\gamma^{+}\left(P_{0}\right)$ runs under $\tilde{\gamma}^{+}\left(P_{0}\right)$. After that $\gamma^{+}\left(P_{0}\right)$ enters into $Q_{3}$ and one of the following two cases occurs:

(c) $\gamma^{+}\left(P_{0}\right)$ meets the negative $x$-axis at a point $P_{3}$;

(d) $\gamma^{+}\left(P_{0}\right)$ goes to infinity without intersecting the negative $x$-axis.

In the case (c), $P_{3}$ is on the left-hand side of $P_{1}$ because $\gamma^{+}\left(P_{0}\right)$ runs under $\tilde{\gamma}^{-}\left(P_{0}\right)$.

To complete the proof, we have to find a neighborhood $U$ of the origin such that all positive orbits of (2.1) starting in $U$ go out of $U$ and its complement $U^{c}$ is a positively invariant set. Taking the uniqueness of solutions and the vector field of (2.1) into account, we can construct a suitable neighborhood $U$ of the origin. We divide our consideration into four kinds of combinations as follows.

Combination of (a) and (c): Consider the region that is enclosed by the arc $P_{2} P_{0} P_{3}$ and the line segment $P_{2} P_{3}$. Then the region is proper for a neighborhood $U$.

Combination of (a) and (d): Let $P_{4}$ be the intersection point of $\gamma^{+}\left(P_{0}\right)$ with the vertical line passing through $P_{2}$. Then we may choose the region that is enclosed by the arc $P_{2} P_{0} P_{4}$ and the line segment $P_{2} P_{4}$ as a neighborhood $U$. 
Combination of (b) and (c): It follows from the property (ii) of (2.1) that $\gamma^{+}\left(P_{0}\right)$ goes through $Q_{2}$ and $Q_{1}$ in order. Since $\gamma^{+}\left(P_{0}\right)$ has no vertical asymptotes, it crosses the line $x=x_{0}$. Let $P_{5}$ be the intersection point. Consider the region that is enclosed by the $\operatorname{arc} P_{0} P_{3} P_{5}$ and the line segment $P_{0} P_{5}$. Then the region is appropriate for a neighborhood $U$.

Combination of (b) and (d): Let $P_{6}$ be any point on the negative $x$-axis and let $P_{7}$ be the intersection point of $\gamma^{+}\left(P_{0}\right)$ with the vertical line passing through $P_{6}$. By the same reason as in the preceding paragraph, $\gamma^{+}\left(P_{6}\right)$ of $(2.1)$ crosses the line $x=x_{0}$. Let $P_{8}$ be the intersection point. Consider the region that is enclosed by the arc $P_{0} P_{7}$ of $\gamma^{+}\left(P_{0}\right)$, the line segment $P_{6} P_{7}$, the arc $P_{6} P_{8}$ of $\gamma^{+}\left(P_{6}\right)$ and the line segment $P_{0} P_{8}$. Then the region is suitable as a neighborhood $U$.

The proof of Lemma 3.2 is now complete.

\section{Existence of limit cycles}

Let us leave the subject of neighborhood of the origin and turn our attention to the asymptotic behavior of orbits of (2.1) in regions that are far from the origin. We present sufficient conditions for system (2.1) to have properties $\left(X_{1}^{+}\right)$and $\left(X_{3}^{+}\right)$and a necessary condition for system $(2.1)$ to have property $\left(X_{2}^{-}\right)$or $\left(X_{4}^{-}\right)$.

As mentioned in Section 3, letting $\phi_{p}(u)=(p G(x))^{1 / p^{*}} \operatorname{sgn} x, v=y$ and $d \tau / d t=g(x) / \phi_{p}(u)$, we can transformed system (2.1) into system (3.1). Noticing that $u=(p G(x))^{1 / p} \operatorname{sgn} x$, we see that the domain of definition of (3.1) is $\{(u, v)$ : $-(p G(-\infty))^{1 / p}<u<(p G(\infty))^{1 / p}$ and $\left.v \in \mathbb{R}\right\}$. If

$$
G(\infty)=G(-\infty)=\infty,
$$

then system (3.1) is defined on the whole $(u, v)$-plane.

Lemma 4.1 Assume (4.1). If there exists $a \lambda<p$ such that

$$
\frac{f(x)}{|g(x)|}(p G(x))^{1 / p^{*}} \geq-\lambda
$$

for $|x|$ sufficiently large, then system (2.1) has properties $\left(X_{1}^{+}\right)$and $\left(X_{3}^{+}\right)$.

To prove Lemma 4.1, we may consider system (3.1) instead of system (2.1). We can carry out the proof of Lemma 4.1 in a similar way to that of Lemma 3.1. Since space is limited, we omit the proof.

When we do not assume condition (4.1), we need some kind of divergence of the function

$$
F(x)=\int_{0}^{x} f(\xi) d \xi
$$

for properties $\left(X_{1}^{+}\right)$and $\left(X_{3}^{+}\right)$. 


\section{Lemma 4.2 If}

$$
\limsup _{x \rightarrow \infty} F(x)=\infty \quad \text { and } \quad \liminf _{x \rightarrow-\infty} F(x)=-\infty
$$

then system (2.1) has properties $\left(X_{1}^{+}\right)$and $\left(X_{3}^{+}\right)$.

Proof. Suppose that system (2.1) fails to have property $\left(X_{1}^{+}\right)$. Then there exists a point $P=\left(x_{0}, y_{0}\right) \in Q_{1}$ such that $\gamma^{+}(P)$ remains in $Q_{1}$. Let $y(x)$ be the solution of $(2.3)$ corresponding to $\gamma^{+}(P)$. Then $y(x)>0$ for $x \geq x_{0}$. Hence, we obtain

$$
\begin{aligned}
& (p-1) \phi_{p^{*}}(y(x))-(p-1) \phi_{p^{*}}\left(y_{0}\right) \\
& \quad=-\int_{x_{0}}^{x} f(\xi) d \xi-\int_{x_{0}}^{x} \frac{g(\xi)}{y(\xi)} d \xi \leq F\left(x_{0}\right)-F(x)
\end{aligned}
$$

for $x \geq x_{0}$. From the first equality in (4.3), we can choose a sequence $\left\{x_{n}\right\}$ such that $F\left(x_{n}\right)$ diverges to $\infty$ as $n \rightarrow \infty$. Hence, we have

$$
0<(p-1) \phi_{p^{*}}\left(y\left(x_{n}\right)\right) \leq(p-1) \phi_{p^{*}}\left(y_{0}\right)+F\left(x_{0}\right)-F\left(x_{n}\right) \rightarrow-\infty \quad \text { as } n \rightarrow \infty,
$$

which is a contradiction. Thus, system $(2.1)$ has property $\left(X_{1}^{+}\right)$. Similarly, from the second equality in $(4.3)$, we see that system $(2.1)$ has property $\left(X_{3}^{+}\right)$. The lemma is proved.

We next give a necessary condition for property $\left(X_{2}^{-}\right)$or $\left(X_{4}^{-}\right)$.

Lemma 4.3 Suppose that

$$
\frac{f(x)}{|g(x)|}(p G(x))^{1 / p^{*}} \geq p
$$

for $x>0$ or $x<0,|x|$ sufficiently large. Then system (2.1) fails to have property $\left(X_{4}^{-}\right)$or $\left(X_{2}^{-}\right)$, respectively.

Proof. We prove only that system (2.1) fails to have property $\left(X_{4}^{-}\right)$if $(4.4)$ is satisfied for $x \geq x_{0}$, where $x_{0}$ is a sufficiently large number. It is enough to show that system (3.1) fails to have property $\left(X_{4}^{-}\right)$. Recall that the function $f_{2}(u)$ in system (3.1) is defined in the interval $\left(-(p G(-\infty))^{1 / p},(p G(\infty))^{1 / p}\right)$ (this interval may be $\mathbb{R})$. Since $f_{2}(u)$ is $f(x)(p G(x))^{1 / p^{*}} /|g(x)|,(4.4)$ becomes

$$
f_{2}(u) \geq p \quad \text { for } u_{0} \leq u<(p G(\infty))^{1 / p},
$$

where $u_{0}=\left(p G\left(x_{0}\right)\right)^{1 / p}$. By way of contradiction, we suppose that system (3.1) has property $\left(X_{4}^{-}\right)$. Let $P=\left(u_{0}, v_{0}\right)$ with $v_{0}<-\phi_{p}\left(u_{0}\right)$ and consider the negative orbit $\gamma^{-}(P)$ of (3.1). Then $\gamma^{-}(P)$ crosses the line segment $\left\{(u, v): u_{0}<u<\right.$ $(p G(\infty))^{1 / p}$ and $\left.v=0\right\}$. Hence, $\gamma^{-}(P)$ must necessarily meet the curve $v=$ 
$-\phi_{p}(u)$ in the region $\left\{(u, v): u_{0}<u<(p G(\infty))^{1 / p}\right.$ and $\left.v<0\right\}$. Let $\left(u_{1}, v_{1}\right)$ be the first intersection point of $\gamma^{-}(P)$ with the curve $v=-\phi_{p}(u)$. Then $u_{0}<u_{1}<$ $(p G(\infty))^{1 / p}$ and $v_{1}<0$. We may regard $\gamma^{-}(P)$ as the solution $v(u)$ of

$$
(p-1) \frac{d}{d u} \phi_{p^{*}}(v)=-f_{2}(u)-\frac{\phi_{p}(u)}{v}
$$

satisfying $v\left(u_{0}\right)=v_{0}$. Noticing that $v\left(u_{1}\right)=-\phi_{p}\left(u_{1}\right)$ and $v(u)<-\phi_{p}(u)<0$ for $u_{0} \leq u<u_{1}$ and using by (4.5), we have

$$
\begin{aligned}
-(p-1)\left(u_{1}-u_{0}\right) & <(p-1) \phi_{p^{*}}\left(v\left(u_{1}\right)\right)-(p-1) \phi_{p^{*}}\left(v\left(u_{0}\right)\right) \\
& =-\int_{u_{0}}^{u_{1}} f_{2}(\xi) d \xi-\int_{u_{0}}^{u_{1}} \frac{\phi_{p}(\xi)}{v(\xi)} d \xi \\
& <-p\left(u_{1}-u_{0}\right)-\int_{u_{0}}^{u_{1}} \frac{\phi_{p}(\xi)}{-\phi_{p}(\xi)} d \xi \\
& =-(p-1)\left(u_{1}-u_{0}\right) .
\end{aligned}
$$

This is a contradiction. Thus, system (3.1) fails to have property $\left(X_{4}^{-}\right)$, and neither has (2.1). This completes the proof of Lemma 4.3.

We are now ready to state our main results of the existence of limit cycles for Eq. (2.1). Combining Lemmas 2.1, 3.2, 4.1-4.3, we get Theorem 4.4 below. We also obtain Theorem 4.5 below by the use of Lemmas 2.3, 3.2, 4.1 and 4.2.

Theorem 4.4 Assume (3.2), (3.9) and (4.4). If either (4.1)-(4.2) or (4.3) holds, then system (2.1) has at least one (stable) limit cycle.

Theorem 4.5 Assume (2.9), (2.10), (3.2) and (3.9). If either (4.1)-(4.2) or (4.3) holds, then system (2.1) has at least one (stable) limit cycle.

Remark 4.6 Under conditions (3.2), (3.9) and either (4.4) or (2.9)-(2.10), if $G(\infty)=\infty, \liminf _{x \rightarrow-\infty} F(x)=-\infty$ and there exists a $\lambda<p$ such that

$$
\frac{f(x)}{|g(x)|}(p G(x))^{1 / p^{*}} \geq-\lambda
$$

for $x>0$ sufficiently large, then system (2.1) has at least one stable limit cycle. Similarly, if $G(-\infty)=\infty, \lim \sup _{x \rightarrow \infty} F(x)=\infty$ and there exists a $\lambda<p$ such that

$$
\frac{f(x)}{|g(x)|}(p G(x))^{1 / p^{*}} \geq-\lambda
$$

for $x<0,|x|$ sufficiently large, system (2.1) has at least one stable limit cycle. 
Finally, we compare our main results with Theorems A and B cited in Section 1. Condition (1.6) implies conditions (3.2) and (3.9), and condition (1.7) implies conditions (2.9) and (4.2). Suppose that the former of condition (1.8) holds. Then, if $G(\infty)=\infty$ (resp., $G(-\infty)=\infty$ ), then the former (resp., latter) of condition (4.4) is satisfied; otherwise, we can derive condition (4.3) from condition (1.9). Moreover, conditions (1.10) and (1.11) imply conditions (4.1) and (2.10), respectively. We therefore conclude that Theorems 4.4 and 4.5 with Remark 4.6 extend Theorems A and B.

\section{References}

[1] R. P. AGARWAL, S. R. GRACE and D. O'REGAN, Oscillation Theory for Second Order Linear, Half-Linear, Superlinear and Sublinear Dynamic Equations, Kluwer, New York, 2002.

[2] I. BIHARI, On the second order half-linear differential equation, Studia Sci. Math. Hungar. 3 (1968), 411-437.

[3] F. BRAUER and J.A. NOHEL, The Qualitative Theory of Ordinary Differential Equations: An Introduction, Dover, New York, 1989.

[4] F. BUCCI, On the existence of periodic solutions for the generalized Liénard's equation, Boll. Un. Mat. Ital. 3-B(7) (1989), 155-168.

[5] A. A. DRAGILEV, Periodic solutions of the differential equation of nonlinear oscillations, Prikl. Mat. Mekh. 12 (1952), 85-88 (in Russian).

[6] Á. ELBERT, A half-linear second order differential equation, Colloq. Math. Soc. János Bolyai 30 (1979), 153-180.

[7] M. FARKAS, Periodic Motions, Springer-Verlag, New York, 1994.

[8] A. F. FILIPPOV, A sufficient condition for the existence of a stable limit cycle for an equation of the second order, Mat. Sbornik N. S. 30(72) (1952), 171-180 (in Russian).

[9] J. P. LASALE and S. LEFSCHETZ, Stability by Liapunov's Direct Method, Academic Press, New York, 1961.

[10] N. LEVINSON and O.K. SMITH, A general equation for relation oscillations, Duke Math. J. 9 (1942), 382-403.

[11] R. MANÁSEVICH and S. SESDZIWY, $p$-Laplacian and Liénard-type equation, Rocky Mountain J. Math. 27 (1997), 611-617.

[12] J. D. MIRZOV, On some analogs of Sturm's and Kneser's theorems for nonlinear systems, J. Math. Anal. Appl. 53 (1976), 418-425. 
[13] G. SANSONE and R. CONTI, Non-linear Differential Equations, Macmillan, New York, 1964.

[14] J. SUGIE, Some criteria of the existence of limit cycles for a planar system of Liénard type, Nonlinear Anal. 11 (1993), 803-814.

[15] G. VILLARI, Periodic solutions of Liénard's equation, J. Math. Anal. Appl. 86 (1982), 379-386.

[16] G. VILLARI, On the existence of periodic solutions for Liénard's equation, Nonlinear Anal. 7 (1983), 71-78.

[17] Y.-Q. YE, et al., Theory of Limit Cycles, Transl. Math. Monographs 66, Amer. Math. Soc., Providence, 1986.

[18] T. YOSHIZAWA, Stability Theory by Liapunov's Second Method, Math. Soc., Japan, Tokyo, 1966.

[19] Z.-F. ZHANG, et al., Qualitative Theory of Ordinary Differential Equations, Transl. Math. Monographs 101, Amer. Math. Soc., Providence, 1992.

Received 16 September 2004; accepted 5 October 2005

Published Online First 13 August 2007

To access this journal online:

http://www.birkhauser.ch 